\title{
Single injection, double acquisition: a double-edged sword?
}

\author{
Ernst E. van der Wall · Yves G. America • \\ Arthur J. Scholte · Jeroen J. Bax
}

Received: 1 July 2008/Accepted: 1 July 2008/Published online: 24 July 2008

(C) The Author(s) 2008

In the clinical arena detection of myocardial viability is currently based on the use of nuclear techniques, which show preserved tracer uptake and metabolism in viable myocardium $[1,2]$. Assessment of myocardial viability and ischemia continues to be an issue in patients with coronary artery disease and left ventricular dysfunction, in particular in patients following a myocardial infarction [3]. Nuclear imaging has long played an important role in this field. Especially PET imaging using 18F-fluorodeoxyglucose (FDG-PET) has been regarded as the metabolic gold standard of tissue viability, which has been supported by a wide clinical experience [4]. Viability assessment using SPECT techniques has gained more wide-spread clinical acceptance than PET, because it is more widely available at lower cost [5]. Moreover, technical advances in SPECT technology such as gated SPECT imaging further improve the diagnostic accuracy of the test [6-9].

During the past decade progress in the field of myocardial perfusion imaging has resulted in a myriad of choices for perfusion imaging protocols, including choices in types of stressor, imaging modality, perfusion tracer, method of analysis, and a wide range of choices of imaging protocols [10-12]. More

E. E. van der Wall ( $₫)$ · Y. G. America .

A. J. Scholte · J. J. Bax

Department of Cardiology, Leiden University Medical

Center, P.O. Box 9600, Leiden, The Netherlands

e-mail: e.e.van_der_wall@lumc.nl recently, the peculiar contribution of gated SPECT in the assessment of myocardial ischemia and viability has been demonstrated, with the potential to evaluate in a single myocardial perfusion study the presence of preserved tracer uptake and the amount of contractile reserve through the acquisition of gated SPECT during inotropic stimulation with dobutamine [13-16]. In 1999, Everaert et al. did already study changes in global and regional left ventricular function in response to dobutamine infusion assessed in 10 healthy volunteers using sequential gated SPECT myocardial perfusion acquisitions [13]. The authors concluded that changes in wall thickness induced by infusion of low-dose dobutamine can be assessed by sequential gated SPECT myocardial perfusion studies. Based on this study it was suggested that the stress gated SPECT protocol proposed in their study might be helpful to distinguish viable from scar tissue in patients with coronary artery disease by demonstrating a preserved inotropic response in hypoperfused myocardium.

In 2000, Narula et al. evaluated dual-isotope, gated SPECT imaging combined with low and high dose dobutamine as a single test for the characterization of various types of altered myocardial dysfunction [14]. They studied 54 patients with ischemic cardiomyopathy using rest and 4 h-redistribution thallium-201 imaging and dobutamine technetium-99m sestamibi SPECT and showed that this new imaging technique allowed the characterization of dysfunctional myocardium as stunned, hibernating, remodeled and 
nonviable. Over the past years, the gated SPECT imaging protocols have been refined [15, 16], and recently a new technique has been developed consisting of three-dimensional registration of CT coronary angiography (CTCA) and ECG-gated myocardial perfusion SPECT [17]. This technique of registration may assist the integration of information from gated SPECT and CTCA and may have clinical application for the diagnosis of ischemic heart disease [17-19].

In the current issue of this journal, Fallahi et al. studied 51 patients using a single injection double acquisition gated SPECT-low dose dobutamine protocol (SIDAGS-LDD), representing a new way to establish the presence of viability and coronary artery disease [20]. In this novel protocol the rest phase of the routine technetium-99m SPECT protocol was substituted by another phase of image acquisition under continuous infusion of low-dose dobutamine immediately after the initial phase of stress imaging. The new protocol was compared to the standard stress/rest gated SPECT protocol (double injection, double acquisition) and the stress-only gated SPECT protocol (single injection, single acquisition). The SIDAGS-LDD protocol demonstrated a good agreement with the standard protocol and proved to be superior to for the assessment of viability in patients following myocardial infarction. The protocol was also superior to the gated imaging stress-only protocol in detecting more defect reversibility. Procedural advantages of the protocol are the absence of the need for a second injection of the tracer, reducing the duration of the imaging study therewith decreasing the costs and radiation burden to both patients and personnel.

Although the novel protocol offers new perspectives for better streamlining imaging procedures, there are several caveats to the protocol (some of them also recognized by the authors). In the present study by Fallahi et al. [20] the main focus was on defect reversibility rather than on functional parameters (wall motion/thickening, left ventricular ejection fraction) implying that only one side of the spectrum of viability has been highlighted. In addition, only the standard stress/rest method allows the true assessment of defect reversibility whereas the single injection protocols have to rely on a 'reversibility equivalent' as a marker of reversibility. Consequently, it remains difficult in the single injection protocols to accurately assess whether a perfusion defect is truly reversible. Next, it has been previously shown that it is useful to employ different imaging strategies depending on the history of the patient [21]. In the study by Schroeder et al. [21] patients without a previous myocardial infarction showed a normal stress SPECT study in almost one-third (32\%) of patients compared to only $4 \%$ in patients with a previously myocardial infarction. As a result, patients with a stress defect at Tc-99m sestamibi/tetrofosmin SPECT imaging should always undergo a resting SPECT study irrespective of the clinical and stress electrocardiographic findings.

A further caveat is that in patients with an earlier myocardial infarction who undergo gated SPECT imaging, left ventricular function (LVEF) post-stress may not always represent true resting left ventricular function [22-25]. In patients with clinically important stress-induced perfusion abnormalities, the LVEF after stress might be significantly lower than the LVEF at rest both with same-day rest-stress and stress-rest imaging protocols. These observations again justify the stratification of patients before starting the gated SPECT study, underscoring that in patients with a previous myocardial infarction the gated acquisition should preferably be performed during the rest study. Lastly, the results of the present study have to be weighed against a true gold standard of viability such as FDG-PET and evaluation postintervention.

Anyway, this interesting single injection, double acquisition gated SPECT imaging protocol opens new avenues to further explore imaging strategies in patient with suspected and known coronary artery disease.

Open Access This article is distributed under the terms of the Creative Commons Attribution Noncommercial License which permits any noncommercial use, distribution, and reproduction in any medium, provided the original author(s) and source are credited.

\section{References}

1. Bax JJ, Lamb H, Dibbets P, Pelikan H et al (2000) Comparison of gated single-photon emission computed tomography with magnetic resonance imaging for evaluation of left ventricular function in ischemic cardiomyopathy. Am J Cardiol 86:1299-1305

2. van der Wall EE, Heidendal GA, den Hollander W, Westera G, Roos JP (1980) I-123 labeled hexadecenoic 
acid in comparison with Thallium-201 for myocardial imaging in coronary heart disease. A preliminary study. Eur J Nucl Med 5:401-405

3. Thygesen K, Alpert JS, White HD, Joint ESC/ACCF/AHA/ WHF Task Force for the Redefinition of Myocardial Infarction (2007) Universal definition of myocardial infarction. Circulation 116:2634-2653

4. Slart RH, Bax JJ, van Veldhuisen DJ, van der Wall EE, Dierckx RA, Jager PL (2006) Imaging techniques in nuclear cardiology for the assessment of myocardial viability. Int J Cardiovasc Imaging 22:63-80

5. Slart RH, Bax JJ, Sluiter WJ, van Veldhuisen DJ, Jager PL (2004) Added value of attenuation-corrected Tc-99m tetrofosmin SPECT for the detection of myocardial viability: comparison with FDG SPECT. J Nucl Cardiol 11:689-696

6. Bavelaar-Croon CD, Pauwels EK, van der Wall EE (2001) Gated single-photon emission computed tomographic myocardial imaging: a new tool in clinical cardiology. Am Heart J 14:383-390

7. Bavelaar-Croon CD, Kayser HW, van der Wall EE et al (2000) Left ventricular function: correlation of quantitative gated SPECT and MR imaging over a wide range of values. Radiology 217:572-575

8. Sciagrà R, Leoncini M (2005) Gated single-photon emission computed tomography. The present-day "'one-stopshop" for cardiac imaging. Q J Nucl Med Mol Imaging 49:19-29

9. America YG, Bax JJ, Dibbets-Schneider P, Pauwels EK, Van der Wall EE (2005) Evaluation of the Quantitative Gated SPECT (QGS) software program in the presence of large perfusion defects. Int J Cardiovasc Imaging 21:519529

10. van Eck-Smit BL, van der Wall EE, Kuijper AF, Zwinderman AH, Pauwels EK (1993) Immediate thallium-201 reinjection following stress imaging: a time-saving approach for detection of myocardial viability. J Nucl Med 34:737-743

11. Groutars RG, Verzijlbergen JF, Tiel-van Buul MM et al (2003) The accuracy of 1-day dual-isotope myocardial SPECT in a population with high prevalence of coronary artery disease. Int J Cardiovasc Imaging 19:229-238

12. Bokhari S, Ficaro EP, McCallister BD Jr (2007) Adenosine stress protocols for myocardial perfusion imaging. J Nucl Cardiol 14:415-416

13. Everaert H, Vanhove C, Franken PR (1999) Effects of lowdose dobutamine on left ventricular function in normal subjects as assessed by gated single-photon emission tomography myocardial perfusion studies. Eur J Nucl Med 26:1298-1303

14. Narula J, Dawson MS, Singh BK et al (2000) Noninvasive characterization of stunned, hibernating, remodeled and nonviable myocardium in ischemic cardiomyopathy. J Am Coll Cardiol 36:1913-1919
15. Kumita S, Cho K, Nakajo H et al (2001) Serial assessment of left ventricular function during dobutamine stress by means of electrocardiography-gated myocardial SPECT: combination with dual-isotope myocardial perfusion SPECT for detection of ischemic heart disease. J Nucl Cardiol 8:152-157

16. Navare SM, Katten D, Johnson LL et al (2006) Risk stratification with electrocardiographic-gated dobutamine stress technetium-99m sestamibi single-photon emission tomographic imaging: value of heart rate response and assessment of left ventricular function. J Am Coll Cardiol 47:781-788

17. Kumita S, Cho K, Nakajo H et al (2006) Clinical applications of ECG-gated myocardial perfusion SPECT. J Nippon Med Sch 73:248-257

18. van Werkhoven JM, Schuijf JD, Jukema JW et al (2008) Anatomic correlates of a normal perfusion scan using 64slice computed tomographic coronary angiography. Am J Cardiol 101:404-405

19. Pundziute G, Schuijf JD, Jukema JW et al (2007) Prognostic value of multislice computed tomography coronary angiography in patients with known or suspected coronary artery disease. J Am Coll Cardiol 49:62-70

20. Fallahi B, Beiki D, Gholamrezanezhad A et al. (2008). Single Tc99m Sestamibi injection, double acquisition gated SPECT after stress and during low-dose dobutamine infusion: a new suggested protocol for evaluation of myocardial perfusion. Int $\mathrm{J}$ Cardiovasc Imaging. doi: 10.1007/s10554-008-9328-y

21. Schroeder-Tanka JM, Tiel-van Buul MM et al (1997) Should imaging at stress always be followed by imaging at rest in Tc-99m MIBI SPECT? A proposal for a selective referral and imaging strategy. Int J Card Imaging 13:323329

22. Johnson LL, Verdesca SA, Aude WY et al (1997) Postischemic stunning can affect left ventricular ejection fraction and regional wall motion on post-stress gated sestamibi tomograms. J Am Coll Cardiol 30:1641-1648

23. Borges-Neto S, Javaid A, Shaw LJ et al (2000) Post-stress measurements of left ventricular function with gated perfusion SPECT: comparison with resting measurements by using a same-day perfusion-function protocol. Radiology 215:529-533

24. Bavelaar-Croon CD, America YG, Atsma DE et al (2001) Comparison of left ventricular function at rest and poststress in patients with myocardial infarction: Evaluation with gated SPECT. J Nucl Cardiol 8:10-18

25. Ramakrishna G, Miller TD, Hodge DO, O'Connor MK, Gibbons RJ (2006) Differences in left ventricular ejection fraction and volumes measured at rest and poststress by gated sestamibi SPECT. J Nucl Cardiol 13:668-674 